\title{
Klinische und experimentelle Onkologie in der Zukunft
}

\author{
K. Mross \\ Klinik für Tumorbiologie an der Albert-Ludwigs-Universität Freiburg
}

\section{Einleitung}

Patienten mit der Hauptdiagnose «Bösartige Erkrankung» haben traditionell eine große Bedeutung für das Patientenaufkommen von Akutkrankenhäusern. Von 14,45 Millionen stationären Krankheitsfällen entfallen 1,6 Millionen (11\%) auf Tumorerkrankungen. An Hochleistungskrankenhäusern wie den Universitätskliniken sowie Kliniken der Maximalversorgung ist der globale Anteil noch höher und beträgt bis zu $30 \%$, in der inneren Medizin erreicht er mit bis zu $45 \%$ ein Maximum. Die Tendenzen sind jeweils steigend, weil sich durch den steigenden Anteil von älteren Menschen in der Gesamtgesellschaft der Anteil von Patienten mit dieser Hauptdiagnose relativ und absolut vergrößert.

Onkologische Medizin ist insbesondere für forschungsorientierte Krankenhäuser von herausragender Bedeutung, da Standarddiagnostik/-therapien und innovative, experimentelle Tumordiagnostik/-therapie parallel betrieben werden müssen, um medizinischen Fortschritt auf diesem Gebiet zu erarbeiten.

Krankenhäuser mit einem so mächtigen Schwerpunkt Onkologie erhalten aber durch diesen hohen Anteil onkologischer Patienten auch ein spezielles Kostenproblem: Hauptkostenverursacher sind die innovativen Medikamente. So kostet eine CMF-Therapie (600/60/600) 8,20 €, eine EC-Therapie (90/600) $308 €$ und DOX/DOC (60/75) $1310 €$ (reine Medikamentenkosten ohne Bereitstellungskosten). Es kommen weitere enorme finanzielle Steigerungen im Laborbereich (Spezialanalysen), und im Bereich der Röntgen-, Sonografie- und Nuklearmedizindiagnostik (unter anderem CT, MRT, PET) hinzu, so dass erhebliche ökonomische Risiken mit der klinischen und experimentellen Onkologie in der Zukunft verknüpft sein werden. Denn

1. sprengt die Kostenentwicklung in der Onkologie die Grenzen der Budgetierung,
2. müssen kostspielige innovative Medikamente und Diagnostika in Hochleistungszentren eingesetzt werden, weil medizinischer Fortschritt sich von diesen Zentren bis zur Basis entwickelt (nicht umgekehrt) und weil sie Hoffnungsträger für die Verbesserung der Therapiemöglichkeiten von Tumorpatienten darstellen und

3. ist die Anwendung von innovativen, zugelassenen Medikamenten in der ersten Phase des Produktlebenszyklus wegen hoher Forschungs- und Entwicklungskosten teuer.

\section{Finanzierung im deutschen Gesundheitssystem}

Seit 1993 werden Krankenhäuser in Deutschland budgetiert, d.h. die Erträge aus stationären Leistungen sind «gedeckelt» und die jährlichen Fortschreibungen orientieren sich primär am Primat der Beitragsstabilität der Krankenkassen und nicht am objektiven Bedarf der einzelnen Krankenhäuser. Mehrleistungen gegenüber diesen Budgets werden extrem degressiv vergütet (10-15\% des eigentlichen Preises), so dass Mehrleistungen nicht zu Mehreinnahmen führen können; Mehrleistung ist somit unerwünscht. Die Nutzung von onkologischen Spezialpflegesätzen ( $§ 13$ Bundespflegesatzverordnung) wird von den meisten Krankenkassen nicht zugelassen. Die Kosten innovativer Krebstherapien sind mit dem Ertragsrahmen normaler Pflegesätze in der Regel nicht finanzierbar, so dass in Krankenhäusern mit normalen Pflegesätzen der Einsatz neuer und/oder teurer Therapieverfahren sehr restriktiv gehandhabt werden muss. Sonderentgelte bzw. Fallpauschalen sind nur im Bereich der autologen und allogenen Blutstammzelltransplantation zur Behandlung von Leukämien und Lymphomen (einem engen, genau definierten Indikationsgebiet) vereinbart.

Die im System des Sozialrechts für Innovationen vorgesehenen Instrumente wie Strukturverträge und Modellvorhaben

PD Dr. Klaus Mross

Klinik für Tumorbiologie an de

Albert-Ludwigs-Universität Freiburg

Breisacherstraße 117, D-79106 Freiburg i. Br. (Deutschland)

Fax +49 7612061832

E-mail mross@tumorbio.uni-freiburg.de 
werden kaum genutzt, weil es wegen der divergierenden Interessen nicht zur notwendigen Verständigung zwischen Krankenkassen, Krankenhäusern und Kassenärztlichen Vereinigungen kommt.

Bisher hat noch kein Krankenhaus teure Krebstherapien als medizinische (und dann auch vom Patienten zu bezahlende) Wahlleistung (wie Einzelzimmer, Chefarztbehandlung usw.) angeboten, dies wäre endgültig und für alle sichtbar die Einführung einer Mehrklassen-Medizin, die das vorhandene Kapital des Patienten berücksichtigt. Das Ideal, alle medizinischen Leistungen für alle versicherten Patienten bei entsprechender Indikation zu erbringen wird sich jedoch aus ökonomischen Gründen nicht umsetzen lassen. Schon unter den jetzigen Bedingungen erfolgt stetig und ständig eine Art Triage durch den behandelnden Arzt, weil die Indikationsstellung genügend Spielräume bietet, sich für (oder gegen) kostspielige Therapien und Diagnostika zu entscheiden. Dies gilt, solange nicht alle Diagnostik- und Therapieentscheidungen in Form von verbindlichen Leitlinien oder Standards so reglementiert sind, dass die Freiheitsgrade des Arztes gegen Null streben. Im Krankenhaus werden die Grenzen der therapeutischen Freiheit schlicht durch das verfügbare Budget gesetzt.

\section{Strukturdefizite}

Die Entwicklung der internistischen Onkologie in den letzten 30 Jahren ist kürzlich mit all ihren Defiziten beschrieben worden [1]. Unstrittig ist, dass Deutschland bezüglich der Behandlungsresultate bei Tumorpatienten in Vergleichen mit anderen Staaten der EU, den USA und Japan viel besser abschneiden könnte, wenn die derzeit verfügbaren Behandlungsmodalitäten optimal genutzt würden. Deutschland liegt bezüglich der Ergebnisqualität onkologischer Behandlung im unteren Drittel [2]. Die Gründe liegen in der mangelhaften Interdiziplinarität bei der Versorgung onkologischer Patienten, mangelhafter bis fehlender Qualitätssicherung und einem «Wildwuchs» onkologischer Kompetenz. Die innere Organisation der Akutkrankenhäuser ist geprägt durch eine medizinisch-fachliche Spezialisierung. Onkologische Patienten finden sich in der hämatologisch/internistisch-onkologischen, der gastroenterologischen und pulmonologischen Abteilung der Klinik für Innere Medizin, in der Klinik für Chirurgie, Gynäkologie oder Radioonkologie sowie in einigen anderen kleineren Abteilungen wie Urologie, Dermatologie, Hals- und Kiefer- und Neurochirurgie. Es gibt jedoch in der Regel keine Einrichtung mit zentraler Steuerung für Diagnostik und Therapie. Die vorherrschenden Regelungen der Privatliquidation sind zusätzlich kontraproduktiv für eine Abteilungs- bzw. Klinik-übergreifende Versorgung des Patienten.

Patientenorientierte klinische Forschung ist in Deutschland sowohl auf akademischer Seite als auch auf Seiten der Industrie laut einer Studie der Boston Consulting Group nur schwach positioniert. Die bedauernswert geringe deutsche
Beteiligung an internationalen onkologischen Kongressen, die niedrige Anzahl von Beiträgen in den führenden onkologischen Zeitschriften und die sehr geringe Beteiligung an internationalen Studiengruppen (z.B. EORTC) ist seit Jahren ein Thema. Dies hängt jedoch mittelbar mit den strukturellen Problemen der Onkologie zusammen, so dass auch hier nur durch die Schaffung leistungsfähiger Zentren eine Besserung eintreten kann. Obgleich Deutschland einer der weltweit bedeutendsten Standorte für die pharmazeutische Industrie ist, spielt es als Forschungs- und Entwicklungsstandort eine immer geringere Rolle. Die 30 führenden globalen pharmazeutischen Unternehmen unterhalten weltweit 130 Forschungsstätten, nur 10 davon in Deutschland, hingegen 16 in England und 53 in den USA [3]. Selbst deutsche Firmen verlagerten ehemals in Deutschland unterhaltene Forschungsstandorte in die USA. Diese Entwicklung erscheint unter Berücksichtigung der Situation der klinischen Forschung konsequent, da die Fortsetzung pharmazeutischer Forschung als patientennahe, klinische Forschung nicht optimal gewährleistet ist.

Strukturelle Defizite in der Onkologie sind schon lange bekannt; mit der Entwicklung von Tumorzentren, in denen nahezu alle Disziplinen in die Versorgung von Tumorpatienten mit eingebunden sind, wurde in der Vergangenheit versucht, diesen Mangel zu kompensieren. Innerhalb von Tumorzentren findet sich, allerdings dezentral, nahezu jegliche notwendige Sach- und Fachkompetenz zur Diagnostik und Therapie von Tumorpatienten. Es treten aber nicht parallel interdisziplinären Diagnostik- und Behandlungsteams in Aktion, sondern der Ablauf einer «Patientenkarriere» erfolgt dezentral und sequentiell. Die Interdisziplinarität beschränkt sich auf ein Diskussionsforum, bei dem schwierige, komplexe, oder sehr seltene Fälle erörtert werden und ein Konsens bezüglich Diagnostik und Therapie erreicht werden soll. Tumorzentren schaffen es jedoch in der Regel nicht, allen Mitgliedern Aktivität abzutrotzen, Tumorregister zu pflegen, Standards zu formulieren, Qualität zu kontrollieren und Verbindlichkeit zu induzieren. Das hohe Lied der Interdisziplinarität und der Kollegialität wird von allen Beteiligten regelmäßig intoniert, es überdeckt jedoch nur strukturelle Defizite und den Mangel regelmäßiger Qualitätskontrolle.

Diese Form der Tumorzentren wurde auf dem Deutschen Krebskongress 2000 vom Kongresspräsidenten vehement kritisiert. Er machte klar, dass ein Tumorzentrum eine Institution sein sollte, in der die Therapie und Versorgung eines onkologischen Patienten nicht davon abhängen sollte, in welche Klinik/Abteilung er zufällig gelangt ist [4].

Will man eine wirklich kompetente, erstklassige Diagnostik und Therapie von Tumorpatienten, müsste man die verschiedenen Gebiete, Schwerpunkte, Teilbereiche aus den einzelnen Disziplinen heraustrennen und zu einem umfassenden, integrierten Krebsdiagnostik-, -behandlungs- und -forschungszentrum (Comprehensive Cancer Center, CCC) zusammenführen. Dies ist nicht identisch mit den sogenannten Tumorzen- 
tren, die an Universitäten gegründet wurden, um dem Gebot der Interdiziplinarität zu entsprechen, die aber trotzdem in ihren alten Strukturen verankert bleiben. Die klinische und experimentelle Onkologie der Zukunft benötigt einen gewaltigen Wandel an Universitäten und Krankenhäusern der Maximalversorgung.

Die Umverteilung von finanziellen Ressourcen im Krankenhaus zu Gunsten der onkologischen Kliniken/Abteilungen scheitert an überkommenden Strukturen. Es gibt in Deutschland nicht ein einziges klinisches Zentrum für Krebsdiagnostik und -therapie oder gar ein umfassendes, führendes Zentrum für Krebsforschung, -diagnostik und -therapie (CCC). Nicht zu übersehen ist, dass Onkologie-untypische Fachabteilungen in Eigenregie vermehrt Chemotherapien durchführen und Oberärzte sich als Spezialisten aus den onkologischen Stammdisziplinen verpflichten. Der Gipfel des diesbezüglichen Wahnsinns dürfte erreicht sein, wenn die Augenärzte das Aderhautmelanom eigenständig mit kompliziertesten, nebenwirkungsreichen Chemo-, Immun- und Zelltherapien behandeln wollten. Die Selbstkoordination der Fachabteilungen/Fachkliniken stößt angesichts solcher Eigeninteressen an Grenzen. Es fehlt an einem anerkannten Konzept für die $\mathrm{Zu}-$ ständigkeitsverteilung von zentraler und fachgebundener Onkologie, ihrer Verbindung und Steuerung.

Zugelassene oder zur Prüfung anstehende Medikamente und Diagnostika, neue Kombinationstherapien, multimodale Therapiekonzepte, neue Therapieintensitäten und das Spektrum an (Neben-)Wirkungen dieser Therapeutika nehmen zu und erfordern an 7 Tagen in der Woche für täglich $24 \mathrm{~h}$ eine auf allen Ebenen hochqualifizierte Umgebung. Das gesamte Ärzteteam muss über ein umfassendes allgemeinmedizinisches und onkologisches Fachwissen verfügen, ebenso wie das Pflegeteam. Parallel sollten analytische Speziallabors permanent nutzbar sein und das radiologische Diagnostikteam muss mit entsprechende Expertise zur Erfolgsbeurteilung einer Antitumorbehandlung beitragen. Ein solches Zentrum müsste übrigens auch an Sonn- und Feiertagen Hochleistungsmedizin liefern können und nicht auf Minimalversorgung heruntergeschaltet werden. Die Forderung lautet daher, Aufbau von echten onkologischen Kompetenzzentren, die im universitären Bereich Diagnostik, Behandlung, Forschung und Lehre umfasst, in Akuthäusern der Maximalversorgung zumindest Diagnostik und Behandlung und eventuell klinische Forschung. Die Präsentation eines Netzwerkes von CCCs und von deren Kooperation kann im Internet unter www.nccn.org angesehen werden.

Die notwendigen Ressourcen zum Aufbau solcher Zentren sollten aus den diversen Abteilungen und Kliniken stammen. Bereits vorhandene Ressourcen müssten dazu neu zusammengesetzt werden, alte Abteilungs- und Klinikstrukturen mit ihrem Fachegoismus und ihrer zunftartigen Abschottung aufgegeben werden. Es ist keine Frage, dass multimodale onkologische Therapiekonzepte interdisziplinär abgearbeitet werden müssen. Damit dies jedoch gut funktioniert, ist unmittelbare räumliche Nähe, sind gemeinsame Arbeitsgruppen, gemeinsame Tumorkonferenzen mit verbindlich umzusetzenden Entscheidungen, Umsetzungs- und Erfolgskontrolle (fachübergreifendes Qualitätsmanagement) und eine exzellente Kommunikationsstruktur notwendig.

Kritisch zu hinterfragen ist für ein onkologisches Kompetenzzentrum die Größe der verschiedenen Schwerpunkte, ihre Gliederung, der Grad ihrer Autonomie sowie die Führungsstrukturen. Keinesfalls ist es sinnvoll, die altbekannten hierarchischen Strukturen komplett zu übernehmen. Ein derart neues Gebilde in der deutschen Kliniklandschaft wird auch neue Wege der Zusammenarbeit und Führung konzipieren müssen, wobei alte Zöpfe durchaus abzuschneiden wären.

Eine weitere strukturelle Besonderheit in Deutschland ist das Monopol der Krankenhäuser für die stationäre und das der niedergelassenen Vertragsärzte für die ambulante Behandlung. Ein großer Teil der onkologischen Patienten kann leider durch die bisher zur Verfügung stehenden Verfahren nicht geheilt werden, der Verlauf der Behandlung eines onkologischen Patienten ist eher gekennzeichnet durch einen ständigen Wechsel von stationären (in onkologischen Fachabteilungen), ambulanten (in Schwerpunktpraxen für Onkologie/Hämatologie) und gering behandlungsbedürftigen Perioden (in Allgemeinarztpraxen). Ziel der medizinischen Onkologen ist es, eine nicht heilbare Krebserkrankung zumindest in eine chronische, behandelbare Krankheit zu überführen mit dem Ziel, die Patienten möglichst lange nicht nur am sondern auch im Leben zu lassen. Die starre Trennung in stationär/ambulant führt zu erzwungenen Arztwechseln mit entsprechenden Problemen. So gibt es erhebliche Probleme beim Datenaustausch und in der Folge unnötige Doppeldiagnostik (z.B.: Patient bringt nur unvollständige Unterlagen, wie bisherige Krankengeschichte, und keine Orginal-Röntgen-Bilder mit, das Beschaffen fehlender Befunde kann manchmal Tage dauern). Ausführliche Arztbriefe sind auf dem ambulanten Sektor eher selten und einheitliche Diagnostik- und Therapiestandards können bisher nicht vorausgesetzt werden. Stationär eingeleitete Therapien werden wegen Budgetproblemen oft nur partiell, manchmal gar nicht weitergeführt - neuerdings auch wegen befürchteter Regressansprüche der Krankenkassen wegen eines zulassungsüberschreitenden Einsatzes von Arzneimitteln («off-label use»). Andere Behandlungen werden wegen pekuniärer Interessen nicht gerne umgesetzt. Z.B. sind repetitive komplexe Infusions-Chemotherapien mit häufiger Arztkonsultation abrechnungstechnisch lukrativer als orale Chemotherapien mit minimalem Überwachungsaufwand und geringer Arztbesuchsfrequenz. Notwendige komplementäre Therapien wie z.B. aus dem psycho-sozial-onkologischen Bereich oder additive Therapien wie z.B. Schmerztherapie müssen ohne Unterbrechung und Qualitätsminderung vom stationären in den ambulanten Teil überführbar sein, gegebenenfalls bei Patienten aus der näheren Umgebung auch unter Erhalt der Patient-Arzt-Bindung oder Patient-Institution-Bindung. 
Die Medikamentenkosten im ambulanten Bereich liegen für CMF bei $70,2 €$, für EC $926 €$ und für DOX/DOC bei $2386 €$ (im Vergleich zu den Medikamentenkosten im stationären Bereich Steigerungen um das 8,6-, 3,0- und 1,8-fache!). Hinzu kommen die Bereitstellungskosten (Apothekenaufschlag) sowie die Abrechnung der medizinischen Leistungen durch den niedergelassenen Arzt, so dass eine ambulant durchgeführte Chemotherapie de facto für EC und DOX/DOC teurer ist als dieselbe Therapie durchgeführt unter stationären Bedingungen. Chemotherapie innerhalb eines multiprofessionellen Behandlungs- und Diagnostikteams (Ärzte, Pflegekräfte mit Onkologieausbildung, Psycho-Onkologen, Schmerztherapeuten, Sozialberater, Ernährungsspezialisten) zu erhalten, dürfte fast immer vorteilhaft sein. Dieser Grad der Professionalität und Kompetenzbreite wird in der Regel von keiner hämato-onkologischen Schwerpunktpraxis erreicht.

\section{Beispiele in Deutschland}

Es gibt in Deutschland kein Krebsdiagnostik- und Behandlungszentrum, das strukturell und inhaltlich die gesamte Onkologie (dies schließt die bösartigen hämatologischen Systemerkrankungen mit ein) umfasst und in einem eigenständigen Zentrum organisiert ist. Damit gibt es allerdings dann ebenfalls nicht «den» Onkologen, sondern nur Teilexperten wie den Hämatologen, den internistischen Onkologen sowie den Strahlentherapeuten, die als Fachärzte mit Prüfungspflicht existieren. Ist die Ausbildung des Strahlentherapeuten noch überschaubar, so wird es für den Hämatologen und den internistischen Onkologen schon schwieriger.

Die Tumorklinik in der Universitätsklinik in Essen war ein hoffnungsvoller Anfang für die internistische Onkologie, zu einem umfassenden onkologischen Zentrum hat sie sich jedoch nicht entwickelt, denn die Abteilung Hämatologie und die Einrichtung für Knochenmark-Stammzelltranplantation sind nicht in die Tumorklinik integriert. Sie sind eigenständige Institutionen, d.h. nicht einmal die eindeutig internistisch geprägten Schwerpunkte sind unter einem Dach vereint, geschweige denn die onkologischen Teilgebiete der chirurgischen Fächer. Eine umfassende Ausbildung zumindest auf dem Gebiet Hämatologie und internistische Onkologie würde in Essen eine Rotation durch drei voneinander unabhängige Institutionen bedeuten.

Das Deutsche Krebsforschungszentrum (DKFZ) in Heidelberg blieb über die Jahrzehnte ein Solitär, sehr stark der Grundlagenforschung verbunden. Die natürlichen Kooperationspartner, die medizinischen Onkologen (aus allen Fachbereichen), die mit klinischer Forschung die sinnvolle Ergänzung darstellen, existieren zwar, sind jedoch weit verstreut in den verschiedenen Universitätskliniken. Dadurch kann nicht organisiert unter einem Dach zusammen geforscht, diagnostiziert und behandelt werden.
Die Klinik für Tumorbiologie (KTB) in Freiburg, eine Modellklinik, mit ihren drei Kompetenzbereichen internistische Onkologie, rehabilitative Onkologie und Forschung auf dem Gebiet der molekularen Onkologie ist ebenfalls weit davon entfernt, in ihrer jetzigen Form einem umfassenden, «ganzheitlichen»Zentrumsgedanken gerecht zu werden. Zusammen mit den entsprechenden Abteilungen/Kliniken des Universitätsklinikums, mit denen die KTB ein «Tumorzentrum» bildet, ist dezentral zwar wiederum alles zur umfassenden Diagnostik, Therapie und Forschung an und mit Tumorpatienten vorhanden, aber eben nicht unter einem Dach. Auch hier wird letztendlich zu wenig gemeinsam beforscht, nicht gemeinsam diagnostiziert und therapiert.

An der Technischen Universität München am Klinikum «Rechts der Isar» ist ein neuer Versuch gestartet worden, die bekannten Strukturdefizite im Onkologiebereich zu verringern [5]. Die Klinik für Strahlentherapie, die III. Medizinische Klinik mit dem Schwerpunkt Hämatologie und internistische Onkologie sowie die Chirurgische Klinik haben sich in einem interdisziplinären Tumortherapie-Zentrum organisiert, um zumindest bei allen Tumorpatienten, für die eine Kombinationstherapie (chirurgische, Strahlen- und medikamentöse Therapie) in Frage kommt, die Therapie vor Beginn interdisziplinär $\mathrm{zu}$ besprechen. Das organisatorische Zusammenführen der genannten drei Kliniken bezieht sich dabei auf eine interdisziplinäre Poliklinik Onkologie, ein interdisziplinäres «Tumorboard», die Bildung von krankheitsorientierten Teams, ein interdisziplinäres Qualitätsmanagement, Aufbau und Pflege eines Intranets für den fachübergreifenden Informationsaustausch sowie die Einrichtung eines Studienzentrums. Integraler Bestandteil der interdisziplinären Kooperation sind ferner das Institut für Röntgendiagnostik, das Institut für Pathologie, die Abteilung für Interventionelle Radiologie und die $\mathrm{Nu}$ klearmedizinische Klinik. Dieser Versuch zeigt, das den Universitätskliniken die Strukturdefizite im Bereich Onkologie sehr wohl bekannt sind, letztendlich ist dieser Versuch der Münchener Kollegen aber ein eher konservativer Weg, der die vorhandenen Klinikstrukturen (und Hierarchien) nicht antastet. Vielleicht kann man diesen Versuch aber auch als einen ersten Schritt hin zu einem alles umfassenden TumortherapieZentrum ansehen, dann ist er alle Anstrengungen wert. Dies kann aber nur ein Anfang sein. - Leider sind Entwicklungen, die nur im Konsens durchgeführt werden dürfen, immer langsam.

\section{Beispiele innerhalb der EU}

In den 1980er Jahren wurde in Spanien erkannt, dass in der Diagnostik, Behandlung und Erforschung von Krebserkrankungen im Vergleich zu den USA und den Beneluxstaaten ein enormes Defizit bestand. Als Konsequenz wurde der «Medical Oncologist» neu als Facharzt eingeführt sowie Diagnostik- 
und Therapiezentren in allen großen Städten geschaffen, am schnellsten in den Universitätsstädten. Das initiale Fehlen ausgebildeter Onkologen wurde innerhalb kurzer Zeit durch ein breit angelegtes «Fellowship»-Programm zur Ausbildung von spanischen Ärzten (Internisten) im Ausland behoben. Die onkologische Forschung hatte bis zu einer Auswertung 1997 ebenfalls Defizite: Zum einen gab es nur wenige forschende Onkologen (im Vergleich zur EU die Hälfte) und diesen standen pro Jahr nur 137800 \$ zur Verfügung. Das war weniger als der EU-Durchschnitt, der bei 372500 \$ liegt und wesentlich weniger als den US Forschern zur Verfügung steht, nämlich 452900 \$. Inzwischen existieren zwei Krebsforschungs- und Behandlungszentren in Madrid und Salamanca und ein weiteres befindet sich in Barcelona in Gründung; insgesamt werden 10 Einrichtungen angestrebt. In Spanien sah der initiale Plan der Regierung eher die optimale Nutzung des Vorhandenen vor als die Neugründung von «Cancer Centers», trotzdem ist der nun begonnene drastische Umbau der spanischen Infrastruktur Ausdruck einer gewollten Verbesserung, nachdem Defizite als solche erkannt worden sind. In Spanien, nicht unbedingt einem Land mit einer großen onkologischen Vergangenheit und auch nicht ein Land mit besseren finanziellen Möglichkeiten als Deutschland, ist das möglich, was hier nicht möglich erscheint: nämlich umfassende, konsequente Veränderungen nach Analyse der Defizite.

In den Niederlanden zeigte Ende der 1980er Jahre eine Analyse der Versorgungssituation onkologischer Patienten, dass etwa ein Drittel aller Patienten eine insuffiziente, meist unterdosierte Chemotherapie erhielten, dass ein Fünftel der Patienten mit Mammakarzinom unter Nebenwirkungen einer mangelhaften Strahlentherapie litten und dass die 5-JahresÜberlebenszeit von Patienten mit Kolonkarzinom in Abhängigkeit von der chirurgischen Klinik, in der sie operiert wurden, zwischen 20 und $60 \%$ schwankte; also bestanden bei allen drei Therapieformen (medikamentös, strahlentherapeutisch und chirurgisch) Defizite. Im Anschluss an diese Analyse wurde in den Niederlanden ein rigoroses Ausbildungsprogramm sowie Weiterbildungsrichtlinien in diesen Fachdisziplinen durchgesetzt. Ferner wurden Behandlungszentren gegründet in denen Strahlentherapie, medizinische Onkologie und Chirurgie interdisziplinär zusammenarbeiten (müssen). Chemotherapien werden von Krankenkassen nur dann erstattet, wenn die Indikation und Durchführung durch qualifizierte medizinische Onkologen erfolgt. Neu erprobte und verbesserte Operationstechniken werden von den chirurgischen Onkologen der Tumorzentren in die peripheren Krankenhäuser vermittelt, mit intensiven Schulungen und einem rigorosen Qualitätsmanagement. Die ambulante Versorgung in Polikliniken ist integraler Teil der Tumorzentren und wird leistungsgerecht und kostendeckend vergütet. Das Resultat dieser Bemühungen ist bekannt: In nahezu allen Statistiken, z.B. über die 5-Jahres-Überlebenszeiten, stehen die Niederlande bei fast allen onkologischen Erkrankungen in Europa an der Spitze [2]. Auch hier zeigt sich, dass aufgrund der entsprechenden
Analysen, die die Defizite der onkologischen Versorgung aufdeckten, die richtigen Veränderungen im System vorgenommen worden sind und damit die Ergebnisqualität enorm gesteigert werden konnte.

\section{Gesundheitsreformen}

Die angekündigte (vorsichtige) Öffnung der Krankenhäuser für eine ambulante Behandlung wurde, obgleich politisch gewollt, bisher nicht realisiert. Somit kann sich die medizinische Onkologie der Krankenhäuser nicht so dem onkologischen Patienten widmen, wie es eigentlich sinnvoll ist. Wenn der Patient aus dem stationären in den ambulanten Bereich wechselt, ist ein Arztwechsel durch das jetzige System zwingend vorgegeben. Das geplante neue Entgeltsystem nach dem GDRG-System soll nur die Behandlung und Betreuung des Patienten im Krankenhaus vergüten. Bei onkologischen Patienten wird die Betreuung aber auch und gerade außerhalb des Krankenhauses stattfinden. Dass insbesondere bei den Übergängen zwischen Krankenhaus- und Ambulanzsektor das deutsche Gesundheitssystem große Mängel aufweist, ist bekannt.

Das DRG-Abrechnungssystem beruht auf der Annahme, dass mittels Haupt- und Nebendiagnosen sowie medizinischer Prozeduren eine DRG-Ziffer berechnet werden kann, die den vorliegenden «Fall» ziemlich genau beschreibt. Ein einheitlicher Erlös bundesweit für eine bestimmte DRG-Ziffer wird Hochleistungsmedizin enorm erschweren, weil es den Trend zur Kostennivellierung der medizinischen Leistungen unterstützt. Nur in den chirurgischen Fächern haben die Krankheitsbilder zumindest zum Teil die notwendige Homogenität, so dass man sie zu Fallgruppen zusammenfassen kann: Die Kosten variieren dann zumindest ungefähr mit den Fallmerkmalen, daher kann in Teilbereichen der chirurgischen Fächer das medizinische Abrechnungswesen mittels G-DRG möglicherweise sinnvoll angewendet werden.

Die komplexen Krankheitsbilder der internistischen Onkologie weisen jedoch diese Homogenität und Variantentreue in Diagnostik, Behandlung und Kosten nicht auf, so dass zumindest die internistisch-onkologischen Abteilungen/Kliniken nicht mit einer sachgerechten Entgeltstruktur rechnen können, wenn, wie geplant, die Abrechnung nach DRG tatsächlich zu 100\% umgesetzt wird. Inwieweit die Prozeduren und Kostenstrukturen bedingt durch Innovationen ständig dem medizinischen Fortschritt angepasst werden können, ist noch eine ganz andere Frage. Interessanterweise gibt es weltweit kein Land, dass versucht hat, zu 100\% nach DRG abzurechnen; in Australien sind es 30-40\%, in den USA 40-50\% und in Österreich ist ein derart kompliziertes System mit $\mathrm{Zu}$ - und Abschlägen (je nach Ausstattung, Ort und Lage des Krankenhaus) entstanden, dass ein Fortschritt im Sinne von Vereinfachung nicht erkennbar ist. Ob das DRG-Abrechnungssystem eine Chance bietet, die Trennung zwischen stationärer und 
ambulanter Versorgung zu überwinden, ist aus heutiger Sicht nicht zu beantworten. Solange aber die ambulante ärztliche Versorgung nach anderen Regeln vergütet wird als das Krankenhaus, ist eine weitere Trennung und ein Auseinanderdriften dieser verschiedenen Welten zu befürchten. Die Schnittstelle muss die tagesklinische bzw. ambulante Behandlung im Krankenhaus bzw. in Krebsdiagnostik- und Behandlungszentren darstellen. Für eine nachhaltige und erfolgreiche Patientenversorgung in der Onkologie ist Qualitätssicherung, -nachweis und -management die Grundlage. Das DRG-Abrechnungssystem kann nur notwendige ökonomische Eckdaten liefern, nicht aber ausreichende Informationen für die Bewertung von Prozess- und Ergebnisqualität von onkologischer Diagnostik und Therapie.

Der Gesetzgeber hat für den stationären Bereich erstmals $\mathrm{Zu}$ gangskontrollen für innovative Diagnose- und Behandlungsmethoden eingeführt. Dies wird die bisher im Rahmen des finanziellen Krankenhausbudgets vorhandene Diagnose- und Therapiefreiheit der Krankenhausärzte tangieren und vielleicht mehr noch als das bisherige Budget die Therapiefreiheitsgrade limitieren. Es ist zu erwarten, dass mit dem Argument Qualitätssicherung sowohl im ambulanten als auch im stationären Sektor erhebliche Einschränkungen der Freiheitsgrade in Bezug auf Diagnostik und Therapie zu erwarten sind, weil die Krankenkassen mit den kodierten Diagnosen und Prozeduren enorme Einsichten in die Patientenströme und die Profile der einzelnen Krankenhäuser erhalten werden. Aus dieser Beschränkung kann nur der Patient entfliehen, der entsprechende Zusatzversicherungspakete zu seiner medizinischen Versorgung aufweist, die es seinem behandelnden Arzt wieder erlauben, mehr Freiheitsgrade hinsichtlich Diagnostik und Therapie für seinen Patienten wahrzunehmen.

Das Argument, die Onkologie verschlinge Unsummen für kostspieligste Chemo-, Immun-, und Zelltherapien ist nicht ganz korrekt. In den Niederlanden werden z.B. 35 Millionen $€$ für Zytostatika ausgegeben, für Protonenpumpen-Inhibitoren jedoch 150 Millionen $€$. Für eine gute onkologische Versorgung ist sicherlich eine gewisse Geldmenge notwendig, die vorhandenen Mittel dürften in Deutschland jedoch bei weitem ausreichen, investieren wir doch in unser Gesundheitssystem innerhalb der EU am meisten Geld. Wird eine «kritische Geldmenge» unterschritten, ist die Versorgungssituation deutlich schlechter, wie die Beispiele England und Dänemark zeigen, die leider die höchsten Mortalitätsraten in der EUROCARE Studie aufwiesen [2]. Der größte Medikamentenausgabenposten wird nicht durch Erst- und Zweitlinien-Therapien hervorgerufen sondern durch Dritt-, Viert- und FünftlinienTherapien (meist im off-label use, also außerhalb der zugelassenen Indikationen), für die $70 \%$ der Gesamtkosten aufgewendet werden. Die wissenschaftliche Evidenz, dass dieser Einsatz sinnvoll ist, wird immer dürftiger, je größer die Zahl der bereits durchgeführten Therapien ist. Gerade für diesen kritischen Bereich fehlen jedoch viele Daten, die für fundierte Therapieentscheidungen erforderlich wären. So ist hier viel
Raum für Entscheide, die dem Empirismus statt gesicherter Erkenntnis entstammen und die oft einem off-label use entsprechen. Genau in dieser Grauzone werden die internistischen Onkologen zunehmend in Begründungsnotstände kommen, insbesondere wenn die Ergebnisqualität therapeutischer Maßnahmen überprüft wird. Pharmazeutische Industrie und Krankenkassen sollten sich in diesem Bereich auf Therapieoptimierungsprüfungen einlassen um Therapieentscheidungen besser begründen zu können. Es bleibt zu hoffen, dass der wirklich gut informierte Patient, nicht nur fragt «was kann man denn noch gegen den Tumor tun», sondern auch kritisch hinterfragt «ist denn die neue Therapie überhaupt sinnvoll». Denn oft ist der Griff des Onkologen in den Therapiesack Ausdruck der Hilflosigkeit, die sich ein Arzt jedoch nicht gerne eingestehen wird.

Der zulassungsüberschreitende Einsatz von Arzneimitteln in der Onkologie kann nicht verhindert werden und wird von den Krankenkassen bezahlt werden müssen [6]. Jedoch ist zu fordern, das nur Kompetenzzentren mit entsprechenden Experten solche Nutzung veranlassen können.

\section{Auswirkungen der Internet-Technologie}

Die Durchführung klinischer Studien ist notwendige Voraussetzung für einen therapeutischen (übrigens auch diagnostischen) Fortschritt in der Onkologie, da neue Therapieprinzipien und Diagnostika nur im Rahmen der gesetzlichen Bedingungen, die durch das Arzneimittel- und Medizinproduktegesetz gegeben sind, entwickelt werden können [7]. Neben den klassischen Zulassungsstudien gibt es jedoch auch einen großen Bedarf an weiteren kontrollierten Studien, die der Therapieoptimierung dienen. Für alle gilt, dass jede klinische Studie durch eine Ethikkommission geprüft werden muss und dass sie allen Vorgaben der «Good Clinical Practice» genügen müssen.

Kaum eine Abteilung/Klinik, die onkologische Patienten betreut, ist so groß, dass Phase-II- oder Phase-III-Studien mit vielen Patienten monozentrisch realisiert werden können. Dadurch ergibt sich zwangsläufig die Notwendigkeit einer Kooperation bzw. Vernetzung mit anderen Behandlungszentren, will man ein Projekt innerhalb eines vernünftigen Zeitrahmens beenden. Phase-I- und auch orientierende Pilotprojekte (Machbarkeitsprüfung) sind die einzigen Studienformen, die wegen der limitierten Anzahl von Patienten unter bestimmten Voraussetzungen monozentrisch realisiert werden können. Die Vorteile einer Behandlung im Rahmen von dezidierten Studienprotokollen, wie sie im Rahmen von multizentrischen klinischen Studien formuliert und ausgearbeitet werden, sind vielfältig: dem Autismus von Behandlungszentren wird vorgebeugt, wenn Studien im Verbund realisiert werden; eine Überwachung des Patienten erfolgt nicht nur in Bezug auf die Antitumoreffektivität, sondern auch in Bezug auf die Nebenwirkungen und den Nutzen für den Patienten; eine Qualitäts- 
beurteilung der Behandlungszentren über die Beurteilung der Dokumentationsqualität und durch Audits wird möglich; Defizite können benannt und korrigiert werden.

Die Resultate von klinischen Studien werden veröffentlicht und stehen somit der Allgemeinheit zur Verfügung. Die Internet-Technologie wird $\mathrm{zu}$ einer drastischen Beschleunigung und allgemeinen Verfügbarkeit der Veröffentlichungen führen, wobei auch bei solchen Veröffentlichungen auf ein Peerreview-Verfahren nicht verzichtet werden kann. Die InternetTechnologie wird auch für die Durchführung von Studien einen Quantensprung darstellen, denn sie führt nicht nur zu einer besseren Studientransparenz sondern auch zu einer besseren Information für Patienten über die vielfältigen Behandlungsoptionen einzelner Kliniken und die Möglichkeiten einer Studienteilnahme. Dadurch kommt es auch zur Verbesserung der Rekrutierung von Patienten für die Teilnahme an Studien und damit auch zur dringend erforderlichen beschleunigten Validierung von Innovationen in der Onkologie. Institutionen mit einer inhaltlich schlechten Internetpräsentation werden im Wettbewerb um Patienten, die sich die Kliniken aussuchen können, Nachteile in Kauf nehmen müssen.

\section{Wünsche für die Zukunft}

Die klinische und experimentelle Onkologie in der Zukunft

1. muss den zunftartigen Egoismus der Fächer, die mit Onkologie befasst sind, überwinden,

2. muss die starre Trennung zwischen stationär und ambulant aufheben,

3. muss sich räumlich und strukturell in «Onkologischen Kompetenzzentren» neu formieren (z.B. als Tumordiagnostik- und -therapie-Zentrum, Comprehensive Cancer Center oder interdisziplinäres Tumortherapie-Zentrum),
4. wird sich untereinander und mit ambulanten Praxen vernetzen müssen, um hochwertige klinische Forschung (epidemiologische Studien, Populations-pharmakologische und -genetische Studien, Medikamentenprüfungen und klinische Studien zur Therapieoptimierung) betreiben $\mathrm{zu}$ können.

5. Sie wird in absehbarer Zeit akzeptieren müssen, dass der Versicherungsstatus des Patienten die Freiheitsgrade des medizinischen Onkologen in Bezug auf Diagnostik und Therapie mit determiniert,

6. sie muss statt trennendem Vokabular, wie internistischer, chirurgischer, gynäkologischer, urologischer oder radiologischer Onkologe, den übergeordneten Begriff des medizinischen Onkologen nutzen (ein Teil des Curriculums der Ausbildung zum medizinischen Onkologen in der inneren Medizin, der Gynäkologie, der Chirurgie usw. sollte bei allen Onkologen gleich sein).

7. Sie sollte sich erinnern, dass TEAM «together each one achieves more» heißt und dass dieser Mehrwert, der sich aus Teamarbeit ergibt, von Patienten erwartet wird und

8. sich dafür einsetzen, dass Krebsgesellschaft und Krebshilfe auch ein TEAM werden.

9. Sie wird mit den vorhandenen Milliarden Euros auskommen müssen, dies wird leichter sein mit neuen Strukturen und

10. sie muss aufpassen, dass sie nicht wie vieles Andere in Deutschland (siehe Bildungssystem mit PISA Studie, Sozialsystem, Arbeitsmarktordnung usw.) dem fröhlichen Mittelmass huldigt. Der Konsens «es darf keine Verlierer geben» hat zur Konsequenz, dass keiner gewinnt. Das Gewinnen überlassen wir offensichtlich gerne den anderen, z.B. unseren Partnern in der EU.

Wie wär's mit einer Trendumkehr?

\section{Literatur}

1 Queißer W: 30 Jahre Internistische Onkologie in Deutschland. Onkologie 2000;23:386-390.

2 Survival in cancer patients in Europe. The EUROCARE-2-study. IARC Scientific Publications 1999: 151

3 Nur noch 10 Forschungsstätten. Deutsches Ärzteblatt 2001;98:2714.
4 Weißbach L: Mit Tradition in die Zukunft. Forum DKG Sonderheft 2000:44-46.

5 Molls M, Siess M, Peschel C, Siewert JR: Interdiziplinäres Tumortherapie-Zentrum. Forum DKG Sonderheft 2000:28-30.
6 Kozianka W, Millarg I: Der zulassungsüberschreitende Einsatz von Arzneimitteln als Leistung der gesetzlichen Krankenkasen. Pharm R 2001;23:236244.

7 Mross K, März W: Klinische Studien: Fundament einer Evidenz-basierten Onkologie - Bestandsaufnahme und Zukunft im Zeitalter des Internet. Onkologie 2001;24(suppl 1):24-34. 